\title{
Expression of 11/-HSD in steroid-induced avascular necrosis of the femoral head
}

\author{
LIN WANG, DENG-KE LUO and ZHEN-YU PAN \\ Department of Orthopaedic Microsurgery and Hand Surgery, Zhongnan Hospital \\ of Wuhan University, Wuhan, Hubei 430071, P.R. China
}

Received November 14, 2012; Accepted March 3, 2013

DOI: $10.3892 / \mathrm{mmr} .2013 .1401$

\begin{abstract}
The aim of the present study was to investigate the association between glucocorticoid receptors and steroid-induced avascular necrosis of the femoral head (SANFH). Healthy New Zealand rabbits were randomly divided into 3 groups ( $n=16 /$ group); the normal (no treatment), control (horse serum injections) and treatment (horse serum and methylprednisolone injections) groups. Methylprednisolone and horse serum injections were used to establish a SANFH model in rabbits; magnetic resonance imaging (MRI) and histopathological analysis were used to evaluate the SANFH rabbit model. Total cholesterol and triglyceride contents in the blood of SANFH rabbits were determined. The protein expression levels of $11 \beta$-hydroxysteroid dehydrogenase type $1(11 \beta-H S D 1)$ and type $2(11 \beta-H S D 2)$ were determined using western blotting. The total cholesterol and triglyceride levels in the blood of methylprednisolone-treated rabbits were significantly increased compared with the control and normal groups, which provides evidence in support of the metabolic disorder theory. Based on the results obtained from western blotting, the expression levels of $11 \beta$-HSD1 protein were increased, whereas the expression levels of $11 \beta$-HSD2 protein were decreased following injection. The results of the present study indicate that $11 \beta$-HSDs are important in the development of SANFH. Furthermore, $11 \beta$-HSDs may be important targets for preventing the development of ANFH in patients treated with steroids, which has a significant reference value for the use of steroids in clinical practice.
\end{abstract}

\section{Introduction}

Steroid-induced avascular necrosis of the femoral head (SANFH), frequently encountered in clinical practice, is a

Correspondence to: Zhen-Yu Pan, Department of Orthopaedic Microsurgery and Hand Surgery, Zhongnan Hospital of Wuhan University, 169 Donghu Road, Wuhan, Hubei 430071, P.R. China E-mail: soloistp@163.com

Key words: 11 $\beta$-hydroxysteroid dehydrogenases, steroid-induced avascular necrosis of the femoral head progressive pathological process. It occurs due to obstruction of the blood circulation in the femoral head induced by the improper use of hormones. Additionally, SANFH is able to induce in situ avascular necrosis and disability in a high proportion of patients. When no treatment is provided to patients, the femoral heads may deform or even collapse, impairing hip joint function and causing permanent disability (1). Currently, the incidence of SANFH is markedly increasing and has the potential to become the leading cause of ANFH. Numerous theories have been suggested in an attempt to explain the development of SANFH, including fat embolism $(2,3)$, intravascular coagulation $(3,4)$, intrafemoral head pressure change $(5)$ and osteoporosis (6). However, the pathological mechanism of SANFH has not yet been fully elucidated (7-9). According to previous studies (10-12), the lipometabolism disorder and intravascular coagulation theories may be used to explain the development of SANFH.

$11 \beta$-hydroxysteroid dehydrogenases (11 $\beta$-HSDs), including $11 \beta$-HSD type 1 (11 $\beta$-HSD1) and type 2 (11 $\beta$-HSD2), are the key metabolic enzymes for catalyzing the interconversion of active glucocorticoids (cortisol and corticosterone) and inert 11-keto forms (cortisone and 11-dehydrocorticosterone) (13). To date, numerous studies have demonstrated that 11 $\beta$-HSD1 and $11 \beta-H S D 2$ affect fat cell and endotheliocyte function (14-17). During fat cell and endotheliocyte dysfunction, an abnormal blood flow in terminal vessels is induced and causes complications, including intravascular coagulation, microcirculation disturbance and vascularembolization, which may lead to femoral head necrosis (17). Therefore, the expression of 11 $\beta$-HSD1 and $11 \beta$-HSD2 may be important in SANFH development.

To the best of our knowledge, no previous studies have investigated the association between $11 \beta$-HSD and SANFH. Results of the present study revealed the correlation between $11 \beta-H S D$ and SANFH, via the determination of $11 \beta-H S D 1$ and $11 \beta$-HSD2 expression in a rabbit model; this may have a significant reference value for the use of steroids in clinical practice.

\section{Materials and methods}

Animals. Healthy adult New Zealand rabbits (weight, 2.6-3.2 kg) were obtained from the Experimental Animal Center of Zhongnan Hospital of Wuhan University (Wuhan, China). All the experimental protocols used in this study were approved by the Animal Care and Use Committee of our Hospital. 
Preparation of the SANFH rabbit model and sample collection. The SANFH rabbit model was prepared according to previously described methods $(1,18)$, with minor modifications. Briefly, 48 healthy New Zealand rabbits were randomly divided into 3 groups ( $n=16 /$ group); the normal (no treatment), control (vehicle only) and treatment groups (SANFH model). Rabbits of the control and treatment groups were injected with horse serum $(10 \mathrm{ml} / \mathrm{kg}$; HyClone Laboratories, Inc., Logan, UT, USA) through an ear vein. After 2 weeks, $6 \mathrm{ml} / \mathrm{kg}$ horse serum was similarly injected once a day for 2 days, followed by an injection of $20 \mathrm{mg} / \mathrm{kg}$ methylprednisolone (Pharmacia and Upjohn Company, Puurs, Belgium) into the abdomen of rabbits in the treatment group twice a week for 2 weeks. Following the methylprednisolone injection, 200,000 units of penicillin was injected into the buttock of each rabbit. In the normal and control groups, an equal amount of saline was injected into the buttock muscle. Three animals from each group were examined using magnetic resonance imaging (MRI) and histopathological analysis 2 weeks after the hormone injection. Additionally, the blood and femur head samples of 3 animals from each group were collected 0 (prior to hormone injection), 2, 4 and 8 weeks after the hormone injection.

MRI. An orthogonal head coil was placed on the anesthetized rabbit, with its center located on the hip joint, and the fast spin echo (SE) was used. $\mathrm{T}_{2}$-weighted imaging $\left(\mathrm{T}_{2} \mathrm{~W}_{\mathrm{I}}\right.$; TR/TE, 2500/74 ms), $\mathrm{T}_{1}$-weighted imaging $\left(\mathrm{T}_{1} \mathrm{~W}_{\mathrm{I}}\right.$; TR/TE, 420/20 ms) and $\mathrm{T}_{2} \mathrm{~W}_{\mathrm{I}}$ fat-suppression sequence $\left(\mathrm{FS}-\mathrm{T}_{2} \mathrm{~W}_{\mathrm{I}}\right)$ were performed twice at the coronal position.

Histopathological analysis. Following the sacrifice of each rabbit, both femoral heads, including the metaphyses and thigh-bones, were removed. The tissue sections were dissected and fixed in $10 \%$ formalin, embedded in paraffin, cut into 5 - $\mu$ m-thick sections, de-paraffinized, rehydrated using standard techniques and stained using hematoxylin and eosin (H\&E). The histopathological changes were evaluated using a microscope (Olympus, Tokyo, Japan).

Determination of total cholesterol and triglyceride contents. The automatic biochemistry analyzer (Advia 1650; Siemens Medical Solutions, Erlangen, Germany) was used to determine the total cholesterol and triglyceride contents in the blood samples of SANFH rabbits.

Western blotting. Total proteins of the femoral head tissue were extracted and equal amounts of proteins $(75 \mu \mathrm{g})$ were separated using sodium dodecyl sulfate-polyacrylamide gel electrophoresis (SDS-PAGE) and blotted on polyvinylidene difluoride (PVDF) membranes. These were probed with anti-HSDB1 and anti-HSDB2 rabbit polyclonal IgG (Wuhan Boster Bio-Engineering Co., Ltd., Wuhan, China) and subsequently with goat anti-rabbit/HRP IgG (Wuhan Boster Bio-Engineering Co., Ltd.), and detected using chemiluminescence. To determine the protein loading, antibodies against $\beta$-actin were used.

Statistical analysis. All the experiments were conducted in triplicate (at least) and the data are presented as the mean \pm standard deviation (SD). The data were evaluated using one-way ANOVA, followed by Dunnett's multiple comparisons test between different groups. The statistical significance of differences was analyzed using SPSS software (SPSS for Windows 15.0; SPSS Inc., Chicago, IL, USA) and $\mathrm{P}<0.05$ was considered to indicate a statistically significant difference.

\section{Results}

MRI examination. The femoral heads of rabbits in the normal and control groups were symmetrical. In FS-T $\mathrm{W}_{\mathrm{I}}$, low fat level signals were observed in the cortex of the femoral head in the normal and control groups. By contrast, MRI in the treatment group revealed a larger articular cavity of the femoral head in rabbits of the treatment group. The high-level $\mathrm{FS}-\mathrm{T}_{2} \mathrm{~W}_{\mathrm{I}}$ signal at the metaphyses indicated that edema was present in the bone marrow in the treatment group (Fig. 1).

Histopathological observations. As shown in Fig. 2, changes in the periosteum, cartilage, trabeculae and hematopoietic organization were observed. The periosteum of the femoral heads in the control and normal groups were smooth, and cartilage cells were arranged in an organized manner. The trabeculae were intact and their arrangement was regular, compact and full. The bone cells in the trabeculae were clearly visible with few empty bone lacunae. There were abundant medullary hematopoietic and small fat cells with a normal morphology. By contrast, the periosteum of the femoral heads in the treatment group was incomplete with partially shed cartilage cells. There were a few thin trabeculae with a disordered texture and a number of trabeculae were broken into fragments. A few spindle-shaped osteoblasts were distributed along the trabeculae. The medullary hematopoietic areas were poorly organized, with fewer cells, a sparse capillary network and partially obstructed blood vessels (Fig. 2).

Levels of total cholesterol and triglycerides in the blood of SANFH rabbits. The total cholesterol and triglyceride levels in the blood of rabbits in the treatment group were significantly higher compared with those of the control and normal groups $(\mathrm{P}<0.01$; Fig. 3). Furthermore, the total cholesterol and triglyceride levels in the blood of rabbits in the treatment group gradually increased following injection until the end of the observation period.

Western blotting. The expression levels of $11 \beta$-HSD1 and $11 \beta$-HSD2 proteins were determined using western blotting (Fig. 4). The expression levels of $11 \beta-H S D 1$ and $11 \beta-H S D 2$ proteins in the control and normal groups maintained a stable level. By contrast, the expression levels of $11 \beta$-HSD1 protein in the treatment group increased 2 weeks after the injection, whereas the expression levels of 11 $\beta$-HSD2 protein in the treatment group decreased 2 weeks after the injection. Additionally, the expression levels of $11 \beta$-HSD1 protein in the treatment group were significantly higher compared with those of the control and normal groups at each time-point after the injection $(\mathrm{P}<0.001)$. However, the expression levels of $11 \beta$-HSD2 protein in the treatment group were significantly lower compared with those of the control and normal groups 

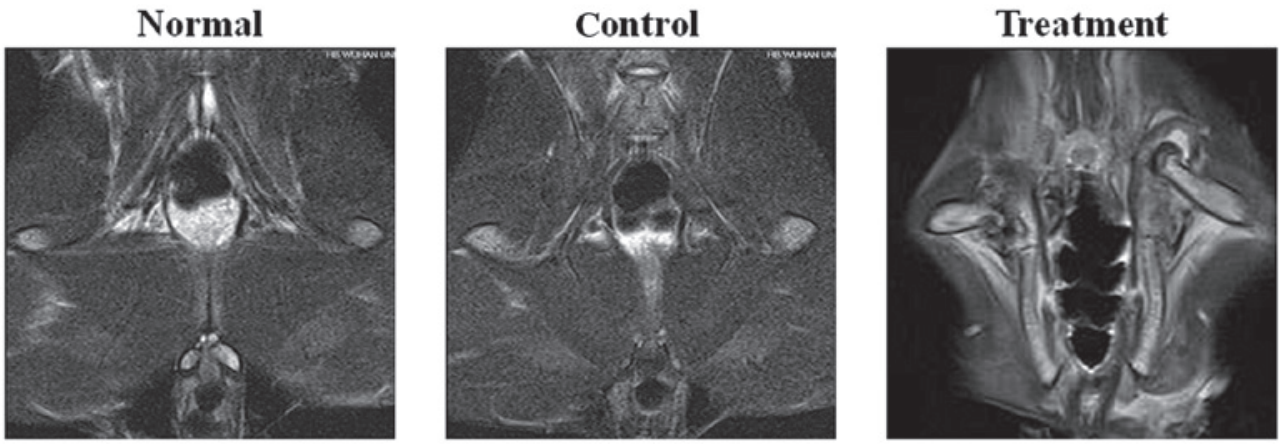

Figure 1. Magnetic resonance imaging (MRI) results showing the femoral heads of the normal, control and treatment groups.
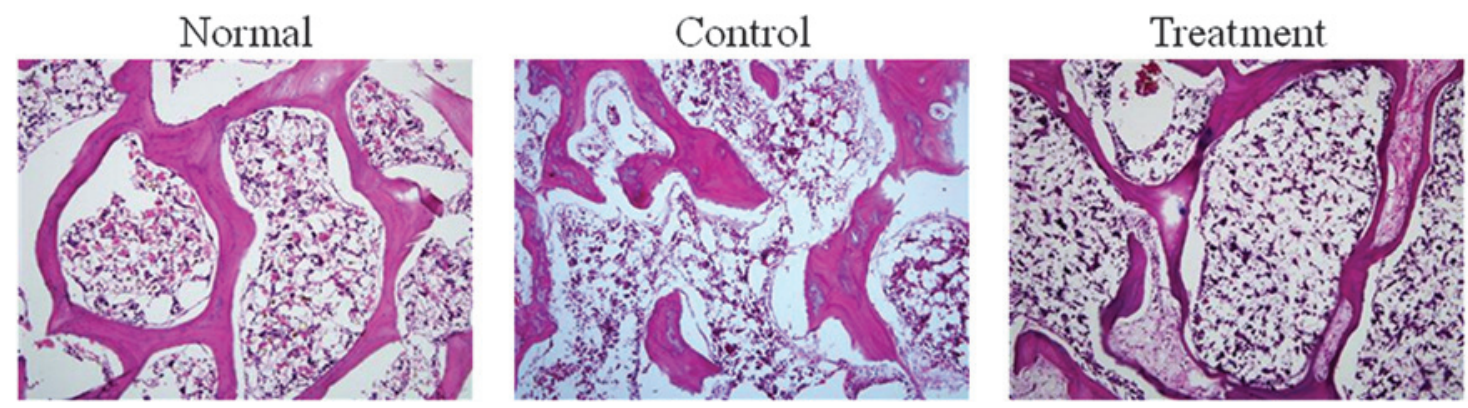

Figure 2. Histopathological examination of tissue sections from the femoral heads of rabbits in the normal, control and treatment groups using hematoxylin and eosin (H\&E) staining (magnification, $\mathrm{x} 100)$.
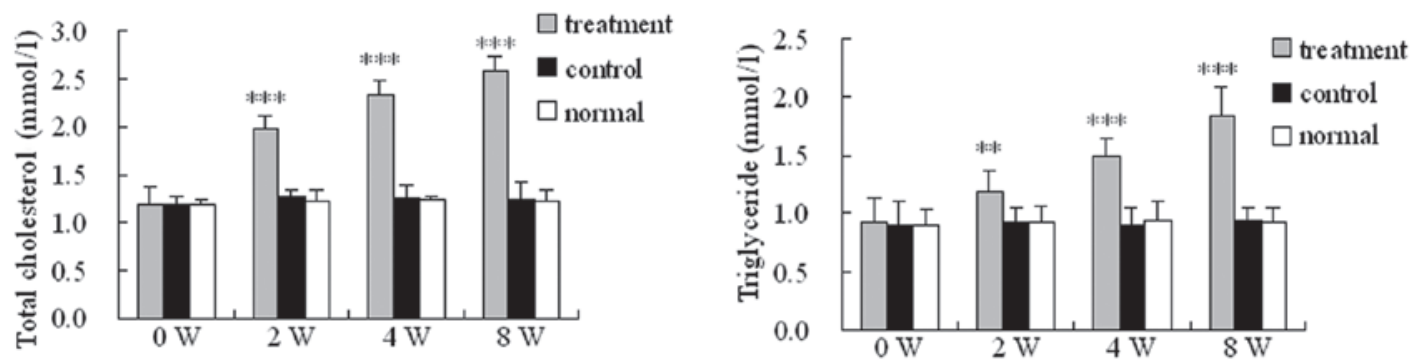

Figure 3. Levels of total cholesterol and triglycerides in the blood of SANFH rabbits. The rabbits were divided into 3 groups ( $\mathrm{n}=10)$ and examined $0,2,4$ and 8 weeks (W) after injection. ${ }^{* *} \mathrm{P}<0.01$ vs. the control groups alone; ${ }^{* * *} \mathrm{P}<0.001$ vs. the control groups alone. SANFH, steroid-induced avascular necrosis of the femoral head.
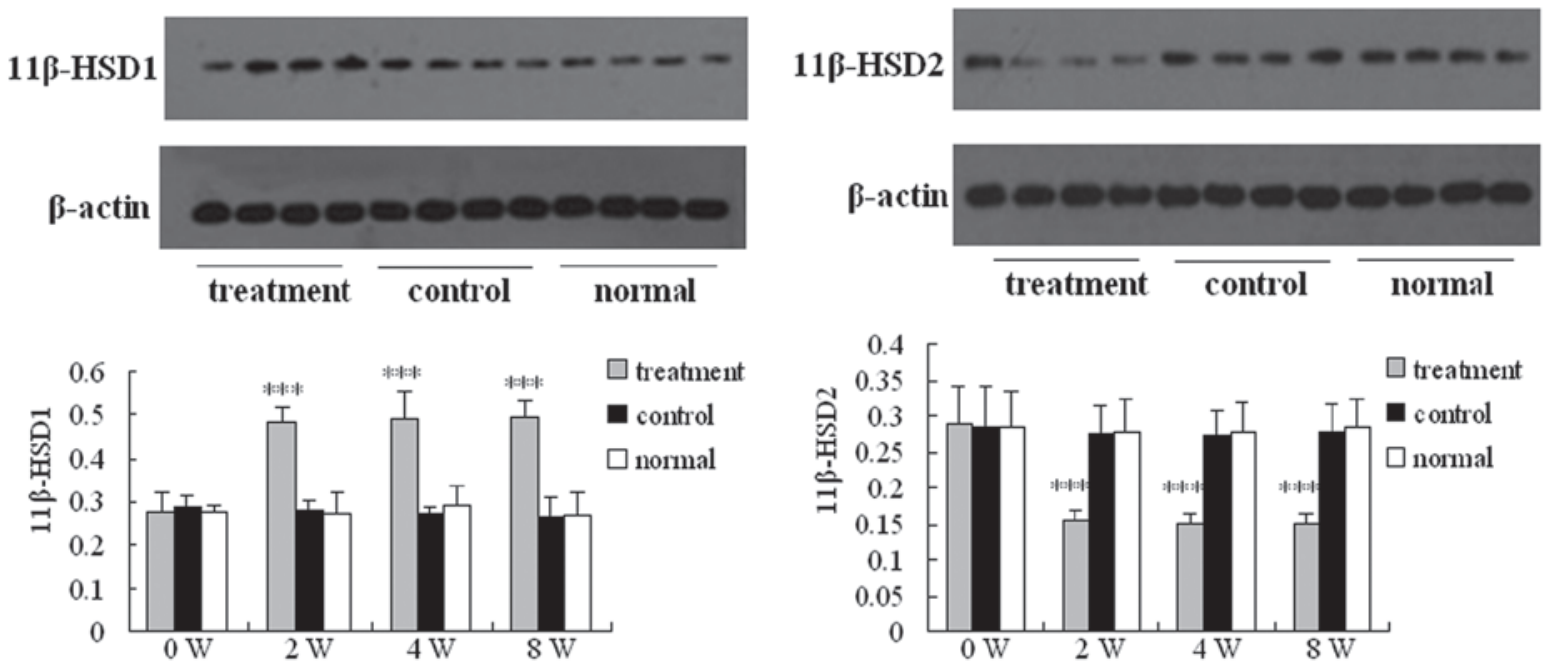

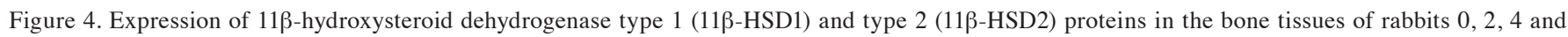
8 weeks (W) after injection. ${ }^{* * * *} \mathrm{P}<0.001$ vs. the control groups alone. 
at each time point after the injection $(\mathrm{P}<0.001)$. The relative protein level was normalized to the intensity of $\beta$-actin, as determined by western blotting.

\section{Discussion}

Steroids have been used in clinical practice to inhibit inflammation, allergy and immune responses in numerous diseases. In 2003, steroids were widely used to treat severe acute respiratory syndrome (19). However, serious adverse reactions may be induced by steroid use, with SANFH being one of the most common. According to the results of previous studies, an increasing number of ANFH cases caused by steriod use have been reported and SANFH often occurs at an age of 30-50 years. Without treatment, hip joint dysfunction occurs in SANFH patients and this disease is considered to be an irreversible process $(20,21)$. Numerous therapeutic methods have previously been used to treat SANFH, including hip arthroplasty, prosthesis and reconstructive surgery of the femoral head, in addition to treatments without surgery; however, these treatments were not particularly effective (22-24). Furthermore, the pathogenesis of SANFH remains unclear. Thus, investigation into the pathogenesis of SANFH and novel therapeutic strategies is urgently required.

In the present study, a rabbit model of SANFH was established to explore potential molecular mechanisms underlying SANFH development. MRI and histopathological analyses were used to evaluate the SANFH rabbit model and these determined that the model had been successfully established and was able to be used for further investigation in this study.

Metabolic disorder has been hypothesized to be the mechanism that underlies SANFH development. Following high hormone intake, the serum concentration levels of lipids, including total cholesterol and triglycerides, are increased, leading to hyperlipidemia. This may cause fat embolism to be induced in the peripheral vessels, leading to intravascular coagulation $(3,7,25)$. Subsequently, fibrin platelets are formed and thrombopoiesis occurs, which induces osteonecrosis via microcirculation disturbance in the femoral head (26-28). Results of the present study demonstrated that the total cholesterol and triglyceride levels in the blood of rabbits treated with methylprednisolone were significantly increased compared with those of the control and normal groups; these results provide evidence in support of the metabolic disorder theory.

According to the results of previous studies, glucocorticoids are able to affect fat metabolism and endotheliocyte function, and may be associated with the necrosis of the femoral head (17). Additionally, glucocorticoids cause serious intramedullary fatty infiltration and induce a diminished blood flow. Complications, including intravascular coagulation, microcirculation disturbance and vascular embolization, are induced by fat cell and endotheliocyte dysfunction, which subsequently lead to ANFH. $11 \beta-H S D 1$ and $11 \beta-H S D 2$ are the two key enzymes that catalyze the conversion of inert 11-keto forms (cortisone) to active cortisol, or vice versa, thus regulating the access of glucocorticoids to steroid receptors (13). Results of the present study showed that the expression levels of $11 \beta$-HSD1 protein were increased, while the expression levels of $11 \beta-H S D 2$ protein were decreased following injection. Therefore, these results indicate that $11 \beta$-HSDs are important in the development of SANFH and that $11 \beta$-HSDs are potentially important targets for preventing the development of ANFH in steroid-treated patients.

\section{References}

1. Wen Q, Ma L, Chen YP, Yang L, Luo W and Wang XN: A rabbit model of hormone-induced early avascular necrosis of the femoral head. Biomed Environ Sci 21: 398-403, 2008.

2. Jones JP Jr: Intravascular coagulation and osteonecrosis. Clin Orthop Relat Res 277: 41-53, 1992.

3. Kawai K, Tamaki A and Hirohata K: Steroid-induced accumulation of lipid in the osteocytes of the rabbit femoral head. A histochemical and electron microscopic study. J Bone Joint Surg Am 67: 755-763, 1985.

4. Nishimura T, Matsumoto T, Nishino $M$ and Tomita K: Histopathologic study of veins in steroid treated rabbits. Clin Orthop Relat Res 334: 37-42, 1997.

5. Wang GJ, Lennox DW, Reger SI, et al: Cortisone-induced intrafemoral head pressure change and its response to a drilling decompression method. Clin Orthop Relat Res 159: 274-278, 1981.

6. Arlet J: Nontraumatic avascular necrosis of the femoral head. Past, present, and future. Clin Orthop Relat Res 277: 12-21, 1992.

7. Jones JP Jr: Fat embolism and osteonecrosis. Orthop Clin North Am 16: 595-633, 1985.

8. Mont MA, Jones LC and Einhorn TA, Hungerford DS and Reddi AH: Osteonecrosis of the femoral head. Potential treatment with growth and differentiation factors. Clin Orthop Relat Res (Suppl 355): S314-S335, 1998.

9. Drescher W, Bünger MH, Weigert K, et al: Methylprednisolone enhances contraction of porcine femoral head epiphyseal arteries. Clin Orthop Relat Res 423: 112-117, 2004

10. Drescher W, Schneider T, Becker C, Hobolth J, Rüther W, Hansen ES and Bünger C: Selective reduction of bone blood flow by short-term treatment with high-dose methylprednisolone: An experimental study in pigs. J Bone Joint Surg Br 83: 274-277, 2001.

11. Feng Y, Yang SH, Xiao BJ, Xu WH, Ye SN, Xia T, Zheng D, Liu XZ and Liao YF: Decreased in the number and function of circulation endothelial progenitor cells in patients with avascular necrosis of the femoral head. Bone 46: 32-40, 2010.

12. Fujioka M, Kubo T, Nakamura F, Shibatani M, Ueshima K, Hamaguchi H, Inoue S, Sugano N, Sakai T, Torii Y, Hasegawa Y and Hirasawa Y: Initial changes of non-traumatic osteonecrosis of femoral head in fat suppression images: bone marrow edema was not found before the appearance of band patterns. Magn Reson Imaging 19: 985-991, 2001.

13. Seckl JR and Walker BR: Minireview: 11beta-hydroxysteroid dehydrogenase type 1- a tissue-specific amplifier of glucocorticoid action. Endocrinology 142: 1371-1376, 2001.

14. Nakano D and Nishiyama A: Programmed $11 \beta$-hydroxysteroid dehydrogenase type 2 reduction: a possible cause of adult-onset disease? J Hypertens 29: 201-203, 2011.

15. Kaur K, Hardy R, Ahasan MM, et al: Synergistic induction of local glucocorticoid generation by inflammatory cytokines and glucocorticoids: implications for inflammation associated bone loss. Ann Rheum Dis 69: 1185-1190, 2010.

16. Kitajima M, Shigematsu M, Ogawa K, et al: Effects of glucocorticoid on adipocyte size in human bone marrow. Med Mol Morphol 40: 150-156, 2007.

17. Kerachian MA, Séguin C and Harvey EJ: Glucocorticoids in osteonecrosis of the femoral head: a new understanding of the mechanisms of action. J Steroid Biochem Mol Biol 114: 121-128, 2009.

18. Matsui M, Saito S, Ohzono K, et al: Experimental steroid-induced osteonecrosis in adult rabbits with hypersensivity vasculitis. Clin Orthop Relat Res 277: 61-72, 1992.

19. Shi B, Li G, Wang P, Yin W, Sun G, Wu Q and Yu G: Effect of antler extract on corticosteroid-induced avascular necrosis of the femoral head in rats. J Ethnopharmacol 127: 124-129, 2010.

20. Jones LC and Hungerford DS: Osteonecrosis: etiology, diagnosis, and treatment. Curr Opin Rheumatol 16: 443-449, 2004.

21. Hungerford DS: Treatment of osteonecrosis of the femoral head: everything's new. J Arthroplasty 22 (Suppl 1): 91-94, 2007.

22. Beris AE, Payatakes AH, Kostopoulos VK, et al: Non-union of femoral neck fractures with osteonecrosis of the femoral head: treatment with combined free vascularized fibular grafting and subtrochanteric valgus osteotomy. Orthop Clin North Am 35: 335-343, 2004. 
23. Nelson CL, Garrison RL, Walz BH and McLaren SG: Resurfacing of only the femoral head - treatment for young patients with osteonecrosis of the femoral head with collapse, delamination and significant head involvement. J Ark Med Soc 100: 162-163, 2003.

24. Asano T, Takahashi KA, Fujioka M, et al: ABCB1 C3435T and G2677T/A polymorphism decreased the risk for steroid-induced osteonecrosis of the femoral head after kidney transplantation. Pharmacogenetics 13: 675-682, 2003.

25. Jones JP Jr and Sakovich L: Fat embolism of bone. A roentgenographic and histological investigation, with use of intra-arterial lipiodol, in rabbits. J Bone Joint Surg Am 48: 149-164, 1966.
26. Jones JP: Epidemiologlcal risk factors for non-traumatic osteonecrosis. Orthopade 29: 370-379, 2000 (In German).

27. Ichiseki T, Matsumoto T, Nishino M, Kaneuji A and Katsuda S: Oxidative stress and vascular permeability in steroid-induced osteonecrosis model. J Orthop Sci 9: 509-515, 2004.

28. Starklint H, Lausten GS and Arnoldi CC: Microvascular obstruction in avascular necrosis. Immunohistochemistry of 14 femoral heads. Acta Orthop Scand 66: 9-12,1995. 